\title{
Electromagnetic interference at the mains ports of an equipment
}

\author{
Mircea Ion Buzdugan, Horia Bălan, Emil E. Simion, Tudor Ion Buzdugan \\ Technical University from Cluj-Napoca, 15, Constantin Daicoviciu street, Cluj-Napoca (Romania), \\ E-mail:mircea.buzdugan@insta.utcluj.ro,horia.balan@eps.utcluj.ro,emil.simion@et.utcluj.ro, \\ tudor.buzdugan@aut.utcluj.ro
}

\begin{abstract}
Distribution line hardware can generate radiofrequency interference (RFI). Such interference can impact the $\mathrm{AM}$ and FM bands as well as VHF television broadcasts.

The paper presents a reversed electromagnetic interference problem, due to the proximity of two AM radio broadcasting stations which injected especially common mode conducted emissions over the maximal limits specified by the national regulations in the public overhead low voltage mains network.

These emissions determined the malfunction of the gas heating centrals Themaclassic Saunier Duval installed in the area. The problem was solved by the retro fitting of an extra EMI filter for the mains network, as presented in the paper.
\end{abstract}

Keywords: electromagnetic interference, conducted immunity, conducted emissions, EMI filter, transfer characteristic

\section{Introduction}

Electromagnetic interference (EMI) is a serious and increasing form of environmental pollution. The threat of EMI is controlled by adopting the practices of electromagnetic compatibility (EMC), which has two complementary aspects: it describes the capacity of electrical and electronic systems to operate without interfering with other systems and also describes the ability of such systems to operate as intended within a specified electromagnetic environment.

Interference can propagate from a "source" to a "victim" via the mains distribution network to which both are connected. This is not well characterized at high frequencies, especially since connected electrical loads can present virtually any RF impedance at their point of connection [2].

\section{A conducted emission problem [3]}

Distribution line hardware can generate radio-frequency interference (RFI). Such interference can impact the AM and FM bands as well as VHF television broadcasts. Most power-line noise is from arcs - arcs across gaps on the order of $1 \mathrm{~mm}$, usually at poor contacts. These arcs can occur between many metallic junctions on power-line equipment.
Arcing generates broadband radio-frequency noise from several $\mathrm{kHz}$ to over $1000 \mathrm{MHz}$. Power-line interference affects lower frequency broadcasts more than higher frequencies. The most common affected from low to high frequencies are: AM radio ( 0.54 to $1.71 \mathrm{MHz})$, low-band VHF TV (channels 2 to 6,54 to $88 \mathrm{MHz}$ ), FM radio (88 to $108 \mathrm{MHz}$ ), high-band VHF TV (channels 7 to 13,174 to $216 \mathrm{MHz}$ ) and UHF (ultra-high frequencies, until about $500 \mathrm{MHz}$ ).

In the situation presented below we have analyzed and solved a severe reverse problem. In the case we have studied there were the AM broadcasting stations which injected conducted RF emissions in the overhead low voltage power lines, noise which affected the operation of certain equipments.

The problem occurred first in a village situated at $25 \mathrm{~km}$ from the city of Cluj-Napoca, Romania, due to the presence in the neighborhood of two radio broadcasting stations (amplitude modulated, with the carrier frequencies $f 1=1152 \mathrm{kHz}, f 2=909 \mathrm{kHz}$, and the corresponding output powers $P 1=400 \mathrm{~kW}, P 2=200 \mathrm{~kW}$ ). Because of the proximity of the stations, the electronic circuitry of the gas heating centrals Themaclassic Saunier Duval (heating power of $24 \mathrm{~kW}$ ), installed in the houses of the village, presented malfunctions, and gave error messages on the interface display.

We have measured the RF noise level injected in the mains network (using the spectrum analyzer HM 5014 and the line impedance stabilization network LISN HM 6050-2, from Hameg Instruments) with the setup presented in figure 1, in which one can see that the LISN connection is reversed.

According Romanian specifications, the maximum allowed RF noise level injected in the public low voltage network in the frequency range $150 \mathrm{kHz}-30 \mathrm{MHz}$, must be situated below $52 \mathrm{~dB} \mu \mathrm{V}$.

Measurements revealed a RFI noise spectrum with levels exceeding $72 \mathrm{~dB} \mu \mathrm{V}(20 \mathrm{~dB} \mu \mathrm{V}$ in plus), at the frequency of the carrier and its odd harmonics, both on the $\mathrm{L}$ and the $\mathrm{N}$ conductors (figure 2). 


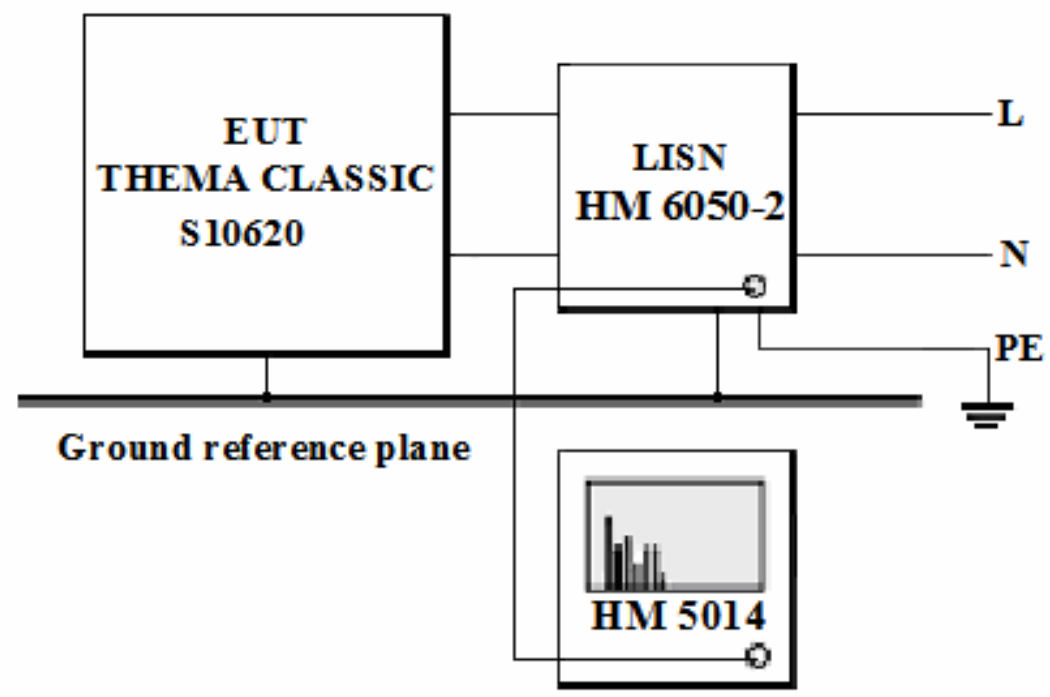

Fig.1. Setup for measuring conducted interference emerging from the overhead mains network.

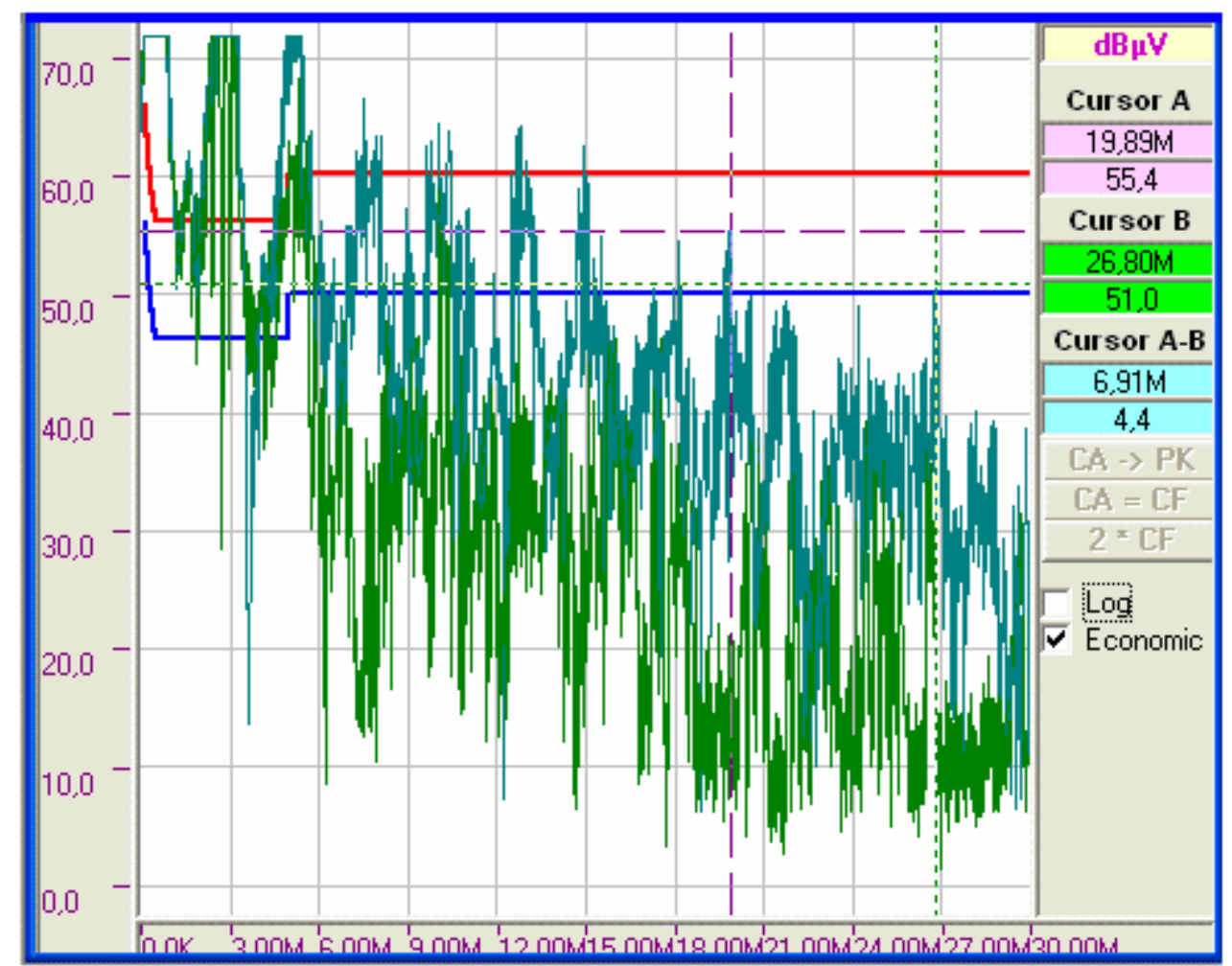

Fig. 2. RF conducted emissions injected on the public mains network by the radio broadcasting stations.

The immunity tests (surge, burst, dips and interruptions) carried out with the immunity testing equipment Best EMC (from Schaffner Instruments) for the electrical circuitry of the gas heating central revealed the conformity with the basic standard for electromagnetic compatibility, EN 61000-4.

The conducted emissions tests measured with a similar setup like the one from figure 1. (in this case the LISN was directly connected), also revealed the conformity with the basic standard for electromagnetic emissions, EN 50011, as it can be seen in figure 3. The noise levels were below the average and the quasi-peak values, according to the standard, in the frequency range of $150 \mathrm{kHz}-30 \mathrm{MHz}$.
Although the gas heating central fulfils the EMC compliance, it can be seen that at the frequency of interest (the carrier frequency of $1 \mathrm{MHz}$ ) the emissions level is only $4 \mathrm{~dB} \mu \mathrm{V}$ lower than the average value specified by the standard.

According to the reciprocity property of the passive filter, emission levels passed through in the low voltage mains network from an equipment represents also the capability of the filter in rejecting the incoming emissions and that slight difference of only $4 \mathrm{~dB} \mu \mathrm{V}$ explain the immunity problem of the gas heating central in the proximity of the radio broadcasting stations. The EMI filter fitted by the manufacturer on the low voltage supply lines of the gas heating central Themaclassic was not enough to insure its immunity in these special noisy conditions. 


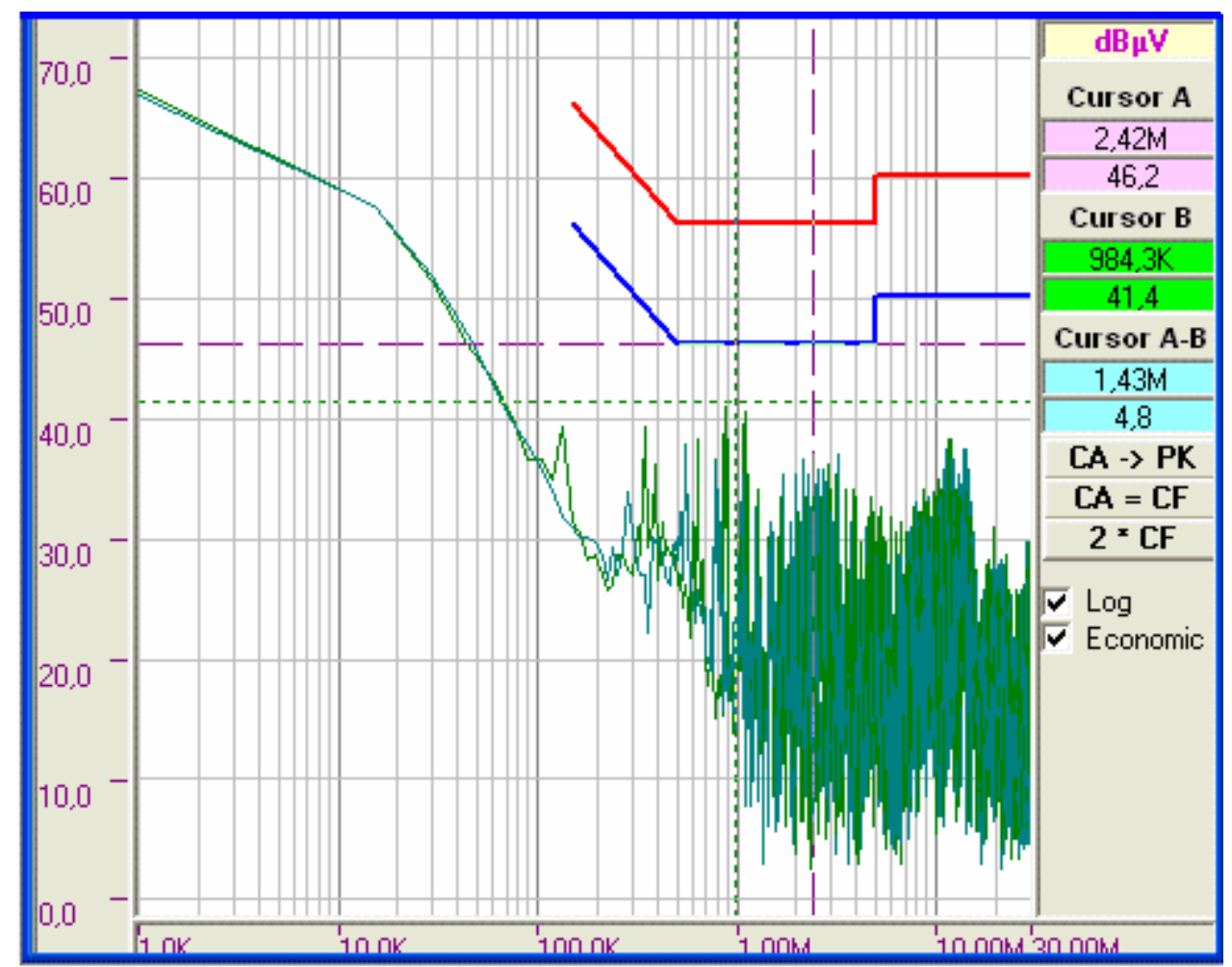

Fig. 3. RF conducted emissions of the gas heating central Themaclassic.

\section{EMI mains filters}

The language spoken by the users of standard or wave filters and EMI filters designers is quite different. The first often speak of poles, zeros, group delay, predistortion, attenuation, and terms such as the order of the filter. On the other hand the EMI filter designers think in terms of attenuation, insertion loss, filter voltage drop, filter voltage rise, and the number of filter sections required to meet the insertion loss.

Mains EMI filters carry potentially high currents at dangerously high voltages, so care is essential in their choice. The working voltage and current rating of components can be decided once the specification is known. The basic specification should also include mechanical details such as the enclosure size, and the limit of weight. The electrical specification should include the voltage and current rating. In addition the EMC performance and the allowable leakage current should be specified. The electrical specification must also comply with national safety standards.

Filters work on the principal of providing a large discontinuity in the characteristic impedance seen by an unwanted signal. The intention is to reflect most of this unwanted energy back to its source. The remaining energy is expended in the inductors through the DC resistance of the coil, the core losses (eddy currents and hysteresis) and the equivalent series resistance of the capacitors. If a filter contains lossy elements, such as a resistor or ferrite component, then the noise energy may be absorbed and dissipated within the filter. If it does not - i.e. if the elements are purely reactive - then the energy is reflected back to its source and must be dissipated elsewhere in the system. This is one of the most important features which distinguish EMI filter design from conventional signal filter design.

Mains filters are tested with a $50 \Omega$ source and load impedance because most RF test equipments have a characteristic impedance of $50 \Omega$. This allows consistent test results and allows direct comparison between one design and another. However, because the source and load impedance in practical situations are not generally $50 \Omega$, the attenuation predicted for a design based on this specification is generally optimistic compared with its performance in working equipment. In the real applications, the source and load impedances, $Z_{S}$ and $Z_{L}$, are complex and in general unknown at the frequencies of interest for suppression [1]. If either or both has a substantial reactive component then resonances are created which may convert an insertion loss into an insertion gain at some frequencies. In the case of mains supplies, the source and load impedance varies widely with frequency. The source impedance is variable over time and can be anywhere from $2 \Omega$ to $2000 \Omega$. The actual impedance is dependent on the loads that are connected to it and the frequency of interest. The characteristic impedance of the mains lead to the load is around $150 \Omega$, and the load itself may have variable impedance. Differential mode impedances may be predictable if the components which make up the source and load are well characterized at RF, but common mode impedances such as are presented by cables or the stray reactance of mechanical structures are essentially unpredictable. Practically, cables have been found to have common mode impedances in the region of $100 \Omega$ to $400 \Omega$, except at resonance, and a figure of $150 \Omega$ is commonly taken for a rule of thumb [2]. 
The typical commercial EMI filters are the balanced $\Pi$ type. Although they seem mainly common mode in appearance, they include components to block both common mode and differential mode components. Such a ready-made filter is F.AM.D.-3600.ZC, manufactured by Arcotronics Ltd., figure 4.
The common mode choke L consists of two identical windings on a single high permeability toroidal core, configured so that differential (line-to-neutral) currents cancel each other. This allows high inductance values, typically $1-10 \mathrm{mH}$, in a small volume without fear of choke saturation caused by the mains frequency supply current. This is because the common mode inductor is wound on ferrite cores having high $\mathrm{A}_{\mathrm{L}}$ values.

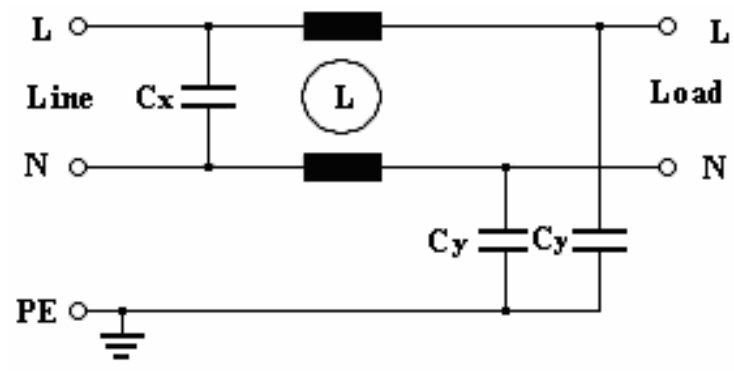

Fig. 4. Typical commercial mains filter.

The full inductance of each winding is available to attenuate common mode currents with respect to earth, but only the leakage inductance will attenuate differential mode interference. The performance of the filter in differential mode is therefore severely affected by the method of construction of the choke, since this determines the leakage inductance. A high leakage inductance will offer greater attenuation, but at the expense of a lower saturation current of the core. Low leakage inductance is achieved by bifilar winding but safety requirements normally preclude this, dictating a minimum separation gap between the windings.

Capacitors CY attenuate common mode interference. The effectiveness of the CY capacitors depends very much on the common mode source impedance of the equipment.
This is usually a function of stray capacitance coupling to earth which depends critically on the mechanical layout of the circuit and the primary-to-secondary capacitance of the mains transformer, and can easily exceed $1000 \mathrm{pF}$. The attenuation offered by the potential divider effect of CY may be no more than $15-20 \mathrm{~dB}$. Some of these filters use the feed-through type of capacitor for better performance. The lead capacitors are less expensive but the self-resonant frequency is lowered by the added lead length.

The common mode choke is the more effective component, and in cases where CY is very severely limited more than one common mode choke may be needed.

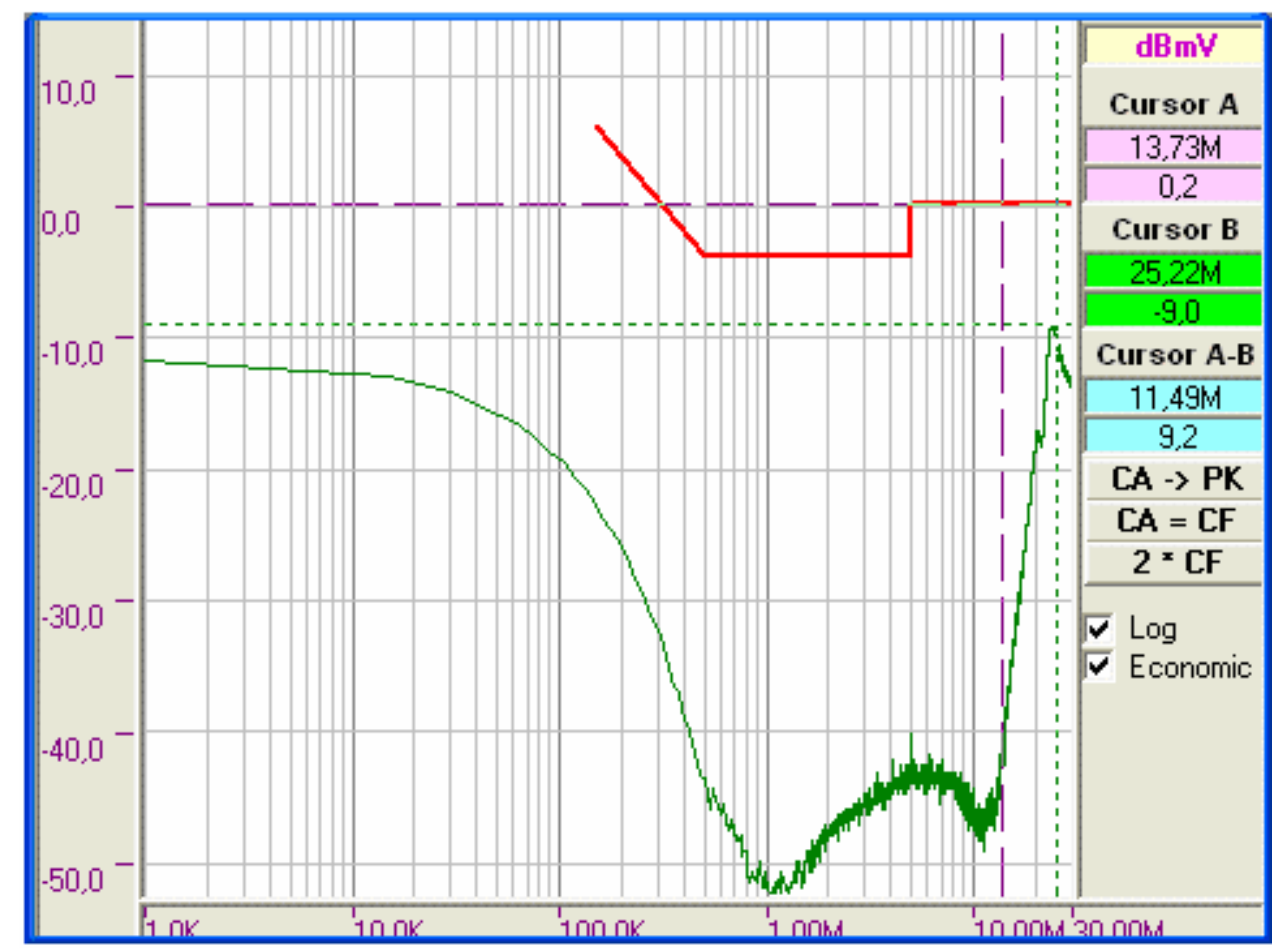

Fig. 5. F.AM.D-.3600.ZC transfer characteristics in differential mode. 


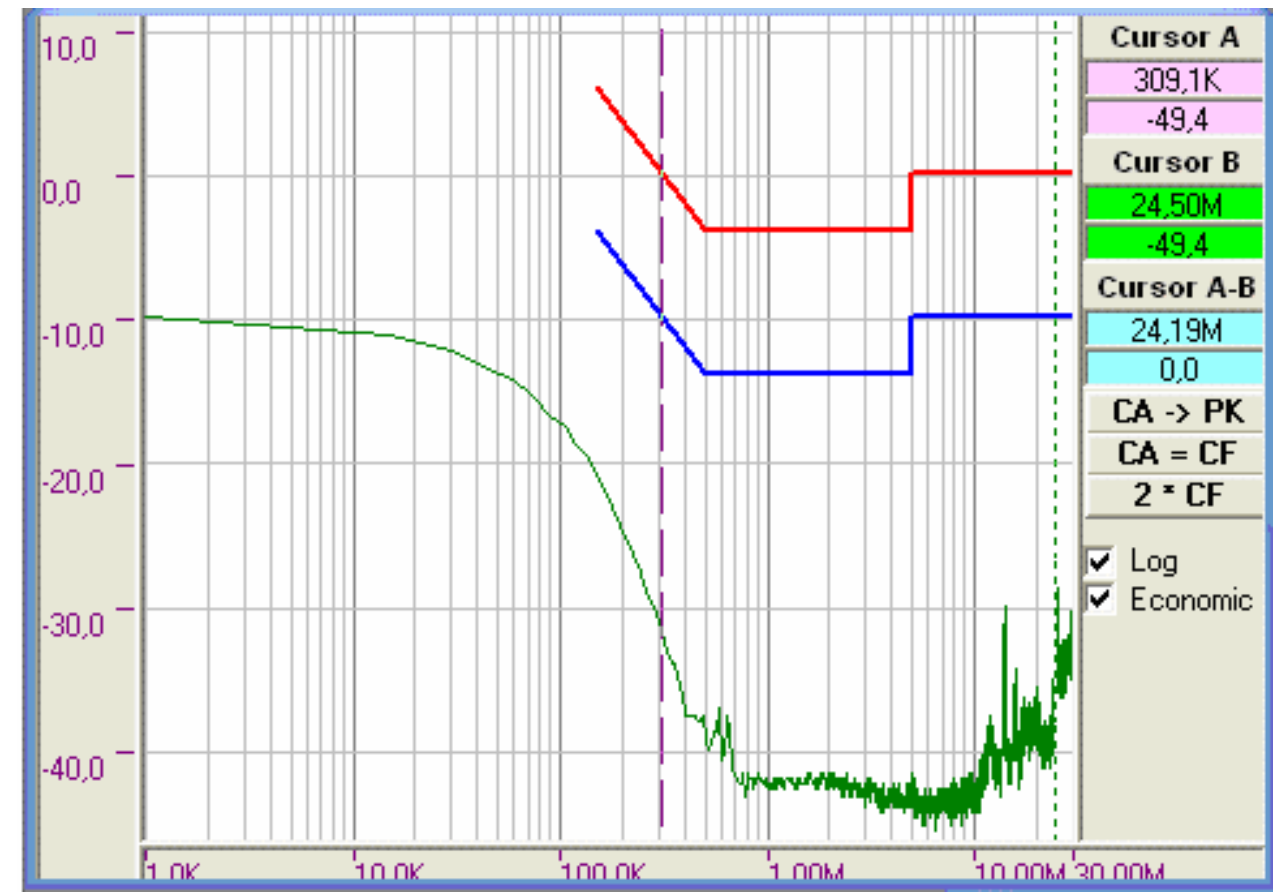

Fig. 6. F.AM.D-.3600.ZC transfer characteristics in common mode.

The CX capacitor attenuates differential mode only, but can have fairly high values, 0.1 to $0.47 \mathrm{mF}$ being typical. $\mathrm{CY}$ is limited in value by the permissible current which may flow in the safety earth, due to the mains operating voltage (or under certain fault conditions). Values for this current, range from $0.25 \mathrm{~mA}$ to $5 \mathrm{~mA}$ depending on the approvals authority, safety class and use of the apparatus.

For the chosen filter in this application, (F.AM.D.3600.ZC, Arcotronics Ltd.), the measured transfer characteristics, both in differential and common mode (using the tracking generator and the spectrum analyzer, from HM 5014, Hameg Instruments), are given in figure 5 . and figure 6 .

The filter parameters are: $\mathrm{CX}=0,6 \mu \mathrm{F}$ (X2 class), $\mathrm{CY}=$ $2 \times 2500 \mathrm{pF}$ (Y2 class), $\mathrm{L}=2 \times 1 \mathrm{mH}$, the leakage current $\mathrm{I}_{\mathrm{L}}=2 \times 0.23 \mathrm{~mA}$ and the temperature range $-25^{\circ} \mathrm{C}$ to $+85^{\circ} \mathrm{C}$. In the frequency range of interest we can see an attenuation of more than $50 \mathrm{~dB}$ in the differential mode and of more than $40 \mathrm{~dB}$ in the common mode.

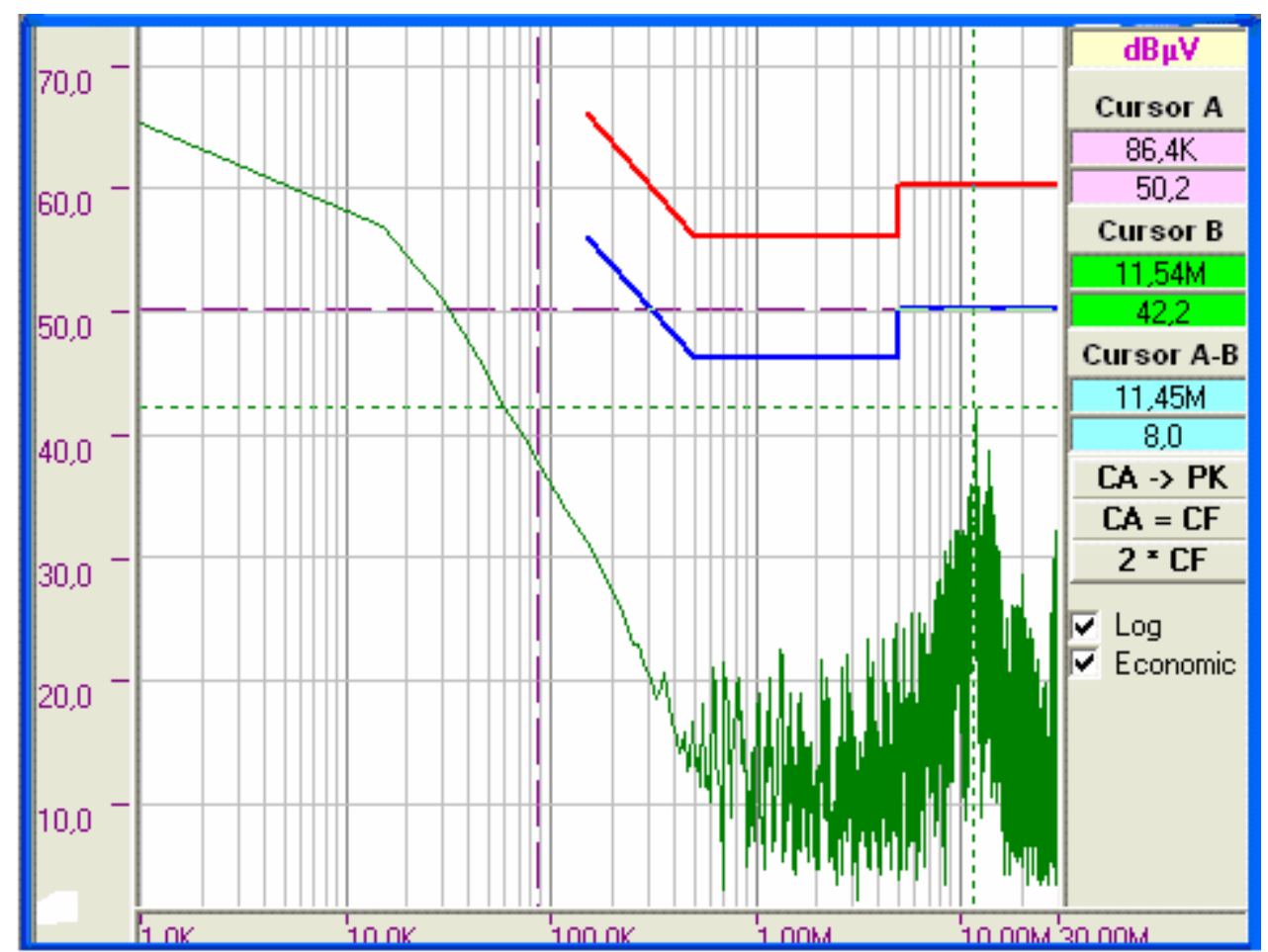

Fig. 7. RF conducted emissions of the gas heating central Themaclassic with the extra EMI filter F.AM.D-.3600.ZC. 
Knowing the reciprocity property of the passive EMI filters, we have once again measured the level of the conducted emissions of the gas heating central with the extra mains filter, and its new emissions level in the frequency range of $150 \mathrm{kHz}-30 \mathrm{MHz}$ is given in figure 7.

Comparing figure 7 with figure 3, we can see a drop in the noise level by $20 \mathrm{~dB} \mu \mathrm{V}$ in the frequency range of interest, which is a good indication that the choice of the filter was the right one.

The installation of this extra EMI mains filter has solved the conducted immunity problem which occurred.

Similar problems have occurred in the proximity of other two radio broadcasting stations in Romania and the solution adopted was the same.

\section{Conclusions}

Some of the reasons for the development and use of block mains filters are [2]:

- Mandatory conducted emission standards concentrate on the mains port

- Safety approvals for the filter have already been achieved
- Many equipment designers are not familiar with RF filter design

In fact, the market for mains filters really took off with the introduction of regulations on conducted mains emissions, compounded by the rising popularity of the switch-mode power supply. With a switching supply, a mains filter is essential to meet these regulations and as we have seen, sometimes, in special conditions, it may be necessary an extra EMI filtering cell.

\section{References}

[1] Winder, S., Analog and Digital Filter Design, Elsevier Science, 2002, pp. 129-132

[2] Williams, T., EMC for Product Designers, Newnes, 2007, pp. 367-373

[3] Buzdugan, M. I., Contributions upon the electromagnetic interference on low voltage mains networks, Ph D Thesis, Technical University of ClujNapoca, Romania, 2007,

[4] Chambers, J., Understanding Common-mode Interference, Westbay Technology, 2004,

[5] Ozenbaugh, R. L., EMI Filter Design, Marcel Dekker, Inc., 2001, pp. 17-25 